\title{
Ultrasonographic Assessment of Passive Cephalic Excursion of Diaphragm During Cough Expiration Predicts Cough Peak Flow in Healthy Adults
}

\author{
Yasuhiro Norisue, Takushi Santanda, Yosuke Homma, Shizuka Tomita, Shinjiro Saito, \\ Jun Kataoka, Yoshihisa Fujimoto, Tadanori Nabeshima, Yasuharu Tokuda, and Shigeki Fujitani
}

\begin{abstract}
BACKGROUND: Evaluation of cough strength is clinically important, especially for patients with neuromuscular disorders and before extubation of mechanically ventilated patients. The pressure gradient between the airway and thoracoabdominal cavities during the cough expiratory phase generates cough flow and passive cephalic movement of the diaphragm. We hypothesized that passive diaphragmatic cephalic excursion, peak velocity, or both during cough expiration might predict cough peak flow (CPF). This physiologic study investigated associations of CPF with simultaneously measured ultrasonographic indices in healthy adults during the cough expiratory phase. METHODS: 56 healthy adults participated in this study. Right hemidiaphragm excursion and peak velocity were measured with ultrasonography during voluntary cough expiration with maximum effort. CPF was simultaneously measured for all coughs along with the ultrasonographic measurements. A linear regression model was used to determine whether ultrasonographic indices predicted CPF. RESULTS: Simple regression analysis showed significant associations between excursion and $\mathrm{CPF}$ in men and women $(P<.001$, beta coefficient 37.8 , 95\% CI 10.9-64.7, adjusted $R^{2}=0.195$ for men; $P<.001$, beta coefficient $46.1,95 \%$ CI 22.3-69.9, adjusted $R^{2}=0.386$ for women). A multiple regression model adjusted for age, height, and sex showed a significant association between $\mathrm{CPF}$ and excursion $(P<.001$, adjusted beta coefficient $38.32,95 \%$ CI $21.20-55.44$, adjusted $R^{2}=0.643$ ). Simple regression analysis showed a significant association between diaphragmatic peak velocity and CPF only in women $\left(P=.004\right.$, beta coefficient $5.07,95 \%$ CI $1.81-8.33$, adjusted $R^{2}=0.280$ for women). CONCLUSIONS: Passive cephalic excursion of the diaphragm during the cough expiratory phase significantly predicted CPF with maximum cough effort in healthy adults. Future studies should investigate the relationship between $\mathrm{CPF}$ and excursion in persons with respiratory and neuromuscular disorders. Key words: cough; respiratory structure and function. [Respir Care 2019;64(11):1371-1376. @ 2019 Daedalus Enterprises]
\end{abstract}

\section{Introduction}

Expiratory muscles are important in producing coughs to protect the airway and lungs. Inability to produce ade-

\footnotetext{
Drs Norisue, Santanda, Homma, Tomita, Kataoka, Fujimoto, and Nabeshima are affiliated with the Department of Emergency and Critical Care Medicine, Tokyo Bay Urayasu Ichikawa Medical Center, Urayasu City, Chiba, Japan. Drs Norisue and Fujitani are affiliated with the Department of Emergency and Critical Care Medicine, St Marianna University Hospital, Kawasaki, Kanagawa, Japan. Dr Saito is affiliated with the Department of Anesthesiology, Jikei University School of Medicine,
}

quate cough increases the risks of aspiration pneumonia and respiratory failure. ${ }^{1-3}$ Therefore, evaluation of expiratory muscle strength is clinically important, particularly in patients with neuromuscular disorders and before extubation of mechanically ventilated patients. Maximum expiratory pressure and cough gastric pressure have been proposed as indices of cough strength in patients with

\footnotetext{
Minato-ku, Tokyo, Japan. Dr Tokuda is affiliated with the Muribushi Okinawa Project for Teaching Hospitals, Okinawa, Japan.
}

The authors have disclosed no conflicts of interest 
neuromuscular disorders.4-7 Voluntary and involuntary cough peak flow (CPF) has also been suggested as an independent predictor of successful extubation of mechanically ventilated patients who have passed a spontaneous breathing trial. ${ }^{8-14}$ Ultrasonographic measurement of cough strength, if feasible, would not require special devices such as gastric manometry or a peak flow meter that must be connected to the endotracheal tube, and would be useful for assessment of cough strength in patients with various medical conditions.

A cough begins with a deep inspiratory phase, followed by intercostal and abdominal muscle contractions with glottic closure. ${ }^{15}$ This results in the compressive phase and an abrupt rise in intrapleural and intra-abdominal pressure. ${ }^{16}$ The glottis then opens, and airway and intrathoracic pressures abruptly decrease. This results in a high pressure gradient between the mouth and thoracoabdominal cavity, which generates sustained flow in the airway to the mouth during a cough. ${ }^{17}$ Although the increase in thoracoabdominal pressure is lower in patients with an endotracheal tube than in non-intubated patients (because of the absence of glottic function), existing evidence indicates that the increase in intra-abdominal pressure during cough is significant and is associated with extubation outcomes. ${ }^{2}$ The higher thoracoabdominal pressure, as compared with pressure in the airway and mouth, results in passive cephalic movement of the diaphragm, which can be detected with ultrasonography. We hypothesized that passive diaphragmatic excursion, peak velocity, or both during the cough expiratory phase might predict CPF. No previous study has investigated the relationship between diaphragmatic movement and simultaneously measured CPF. The aims of this physiologic study were to identify any associations of ultrasonographic indices with diaphragmatic movement and simultaneously measured $\mathrm{CPF}$, and to ascertain normal values for ultrasonographic indices in healthy adults, which may be useful for future studies of patients with various medical conditions.

\section{Methods}

\section{Participants}

Fifty-six healthy Japanese medical students, residents, and critical care fellows (age 23-43 y) participated in this study. The demographic characteristics of the participants

\footnotetext{
Correspondence: Yasuhiro Norisue MD, Department of Pulmonary and Critical Care Medicine, Tokyo Bay Urayasu Ichikawa Medical Center, 3-4-32 Todaijima Urayasu City, Chiba, Japan 2790001. E-mail: norisue.yasuhiro@gmail.com.
}

DOI: $10.4187 /$ respcare .06780

\section{QUICK LOOK}

\section{Current knowledge}

Evaluation of cough strength is clinically important. Conventional measures of cough strength include cough peak flow, vital capacity and respiratory muscle pressures.

\section{What this paper contributes to our knowledge}

This study shows that passive cephalic excursion of the diaphragm during the cough expiratory phase significantly predicted cough peak flow with maximum cough effort in healthy adults.

are shown in Table 1. Written informed consent was obtained from all participants, and the study was approved by the Institutional Review Board of Tokyo Bay Urayasu Ichikawa Medical Center.

\section{Observations and Measurements}

Subjects in a supine position were instructed to produce 6 coughs with maximum effort. CPF was measured during all coughs with a peak flow meter (PersonalBest, Philips Healthcare, Andover, Massachusetts). A CX50 ultrasound device (Philips) was used to assess ultrasonographic indices of the diaphragm with a sector transducer $(3.5 \mathrm{MHz})$. The transducer was positioned on the abdominal wall just below the lowest right rib, between the midaxillary line and mammillary line in the longitudinal scanning plane to the cephalic direction, with the liver as an acoustic window (Fig. 1). ${ }^{18-20}$ The angle of the transducer was adjusted so that the ultrasound beam was perpendicular to the posterior third of the right hemidiaphragm. ${ }^{21}$ The subject was then instructed to cough into the peak flow meter. Because diaphragm excursion and diaphragm peak velocity cannot be measured simultaneously, diaphragm excursion was measured during the first 3 coughs, and diaphragm peak velocity was measured during the last 3 coughs. To measure excursion, the M-mode interrogation line was adjusted to ensure that it was perpendicular to the movement of the posterior third of the right hemidiaphragm. ${ }^{20} \mathrm{Ex}$ cursion was measured on the vertical axis of the M-mode and was traced from the end of inspiration to the end of cough expiration (Fig. 2). Peak velocity was measured by placing the tissue Doppler imaging cursor at the posterior third of the right hemidiaphragm (Fig. 3). Three intensivists with at least 3 y of experience in diaphragm ultrasonography performed the evaluations.

\section{Statistical Analysis}

The mean excursion and peak velocity of 3 coughs and simultaneously measured $\mathrm{CPF}$ were used to analyze cor- 
Table 1. Demographic Variables of Participants

\begin{tabular}{lc}
\hline \hline Subjects, $n$ (\% male) & $56(55)$ \\
Age, y & $32 \pm 5$ \\
Height, cm & $165 \pm 9$ \\
Weight, kg & $60 \pm 13$ \\
\hline Data are presented as mean \pm SD or $n(\%)$. & \\
\hline
\end{tabular}

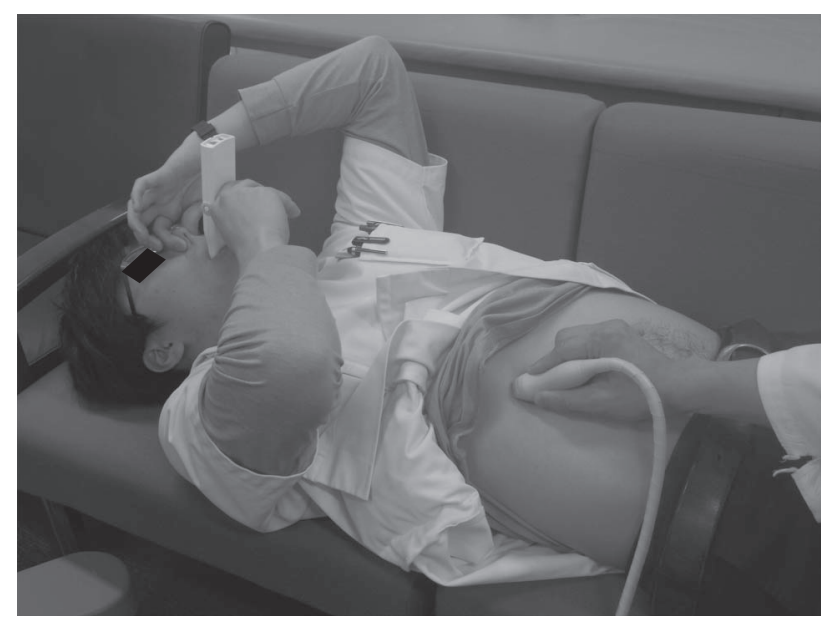

Fig. 1. Simultaneous measurement of cough peak flow and ultrasonographic indices.

relations of $\mathrm{CPF}$ with excursion peak velocity. Pearson coefficients were used to estimate correlations between excursion and peak velocity. To determine whether ultrasonographic indices predicted CPF, regression models with $\mathrm{CPF}$ as a dependent variable were constructed with diaphragm excursion and peak velocity as the independent variables. If a model showed a significant association between an independent variable and CPF, an adjusted regression model was constructed with age, sex, and height. A Bland-Altman plot was constructed to analyze agreement between measured and predicted CPF as determined with ultrasonographic indices. A 2-tailed $P$ value of $<.05$ was considered to indicate statistical significance. STATA version 14 (Stata Corp, College Station, TX) was used for all statistical analyses except for construction of the Bland-Altman plot, which was done with R3.3.3 (R Foundation for Statistical Computing, Vienna, Austria).

\section{Results}

CPF and ultrasonographic measurements are presented as mean $\pm \mathrm{SD}$ in Table 2 . The associations of CPF with diaphragm excursion and peak velocity are shown in Figures 4 and 5, respectively. The Pearson coefficient was $0.496(P<.001)$ for the correlation between excursion and CPF and $0.347(P<.001)$ for the correlation between peak velocity and $\mathrm{CPF}$. A simple regression model with $\mathrm{CPF}$ as the dependent variable in relation to excursion showed significant associations between excursion and CPF in men and women $(P<.001$, beta coefficient 37.8, 95\% CI 10.9-64.7 for men; $P<.001$, beta coefficient 46.1 , 95\% CI 22.3-69.9 for women). A simple regression model with $\mathrm{CPF}$ as the dependent variable in relation to peak velocity showed a significant association between excursion and $\mathrm{CPF}$ only in women $(P=.78$, beta coefficient $-0.33,95 \%$ CI -2.74 to 2.08 for men; $P=.004$, beta coefficient 5.07 , 95\% CI 1.81-8.33 for women). A multiple regression model adjusted for age, height, and sex showed a significant association between $\mathrm{CPF}$ and excursion $(P<.001$, adjusted beta coefficient 38.32, 95\% CI, 21.2055.44 , adjusted $\mathrm{R}^{2}=0.643$ ). The equation to predict $\mathrm{CPF}$ was

$$
\begin{aligned}
& \text { Predicted } \mathrm{CPF}=\text { diaphragm excursion }(\mathrm{cm}) \times 38.3+ \\
& \qquad \begin{array}{r}
\text { age }(\mathrm{y}) \times 4.42+\text { height }(\mathrm{cm}) \times 0.796+ \\
\operatorname{sex} \times 119-112
\end{array}
\end{aligned}
$$

where male $\operatorname{sex}=1$ and female sex $=0$.

Agreement of measured $\mathrm{CPF}$ with $\mathrm{CPF}$ predicted by diaphragm excursion, age, height, and sex was assessed with a Bland-Altman plot (Fig. 6).

\section{Discussion}

This study shows that diaphragm excursion during cough expiration significantly predicted CPF produced with maximum cough effort. This result is consistent with a previous study where diaphragm excursion values during cough were lower for subjects after a stroke with dysphagia than for healthy volunteers, which suggests a relationship between cough strength and excursion. ${ }^{18}$ This study is the first to investigate direct associations between CPF and simultaneously measured ultrasonographic variables during the cough expiratory phase.

Cough strength is determined mainly by abdominal expiratory muscle strength, which can be evaluated by measuring intra-abdominal pressure (gastric pressure or bladder pressure) during cough. ${ }^{2,5}$ The pressure gradient between the thoracoabdominal cavity and the airway generates cough flow to the mouth, as well as passive cephalic movement of the diaphragm. Previous studies reported that diaphragm excursion was positively correlated with inspiratory volume. ${ }^{22,23}$ Our result suggests that cough inspiratory volume is an important component in generating adequate $\mathrm{CPF}$ and that diaphragm excursion is therefore closely associated with $\mathrm{CPF}$. The marked improvement in predictive accuracy of diaphragmatic excursion after adjust- 


\section{Diaphragm Excursion and Cough Strength}

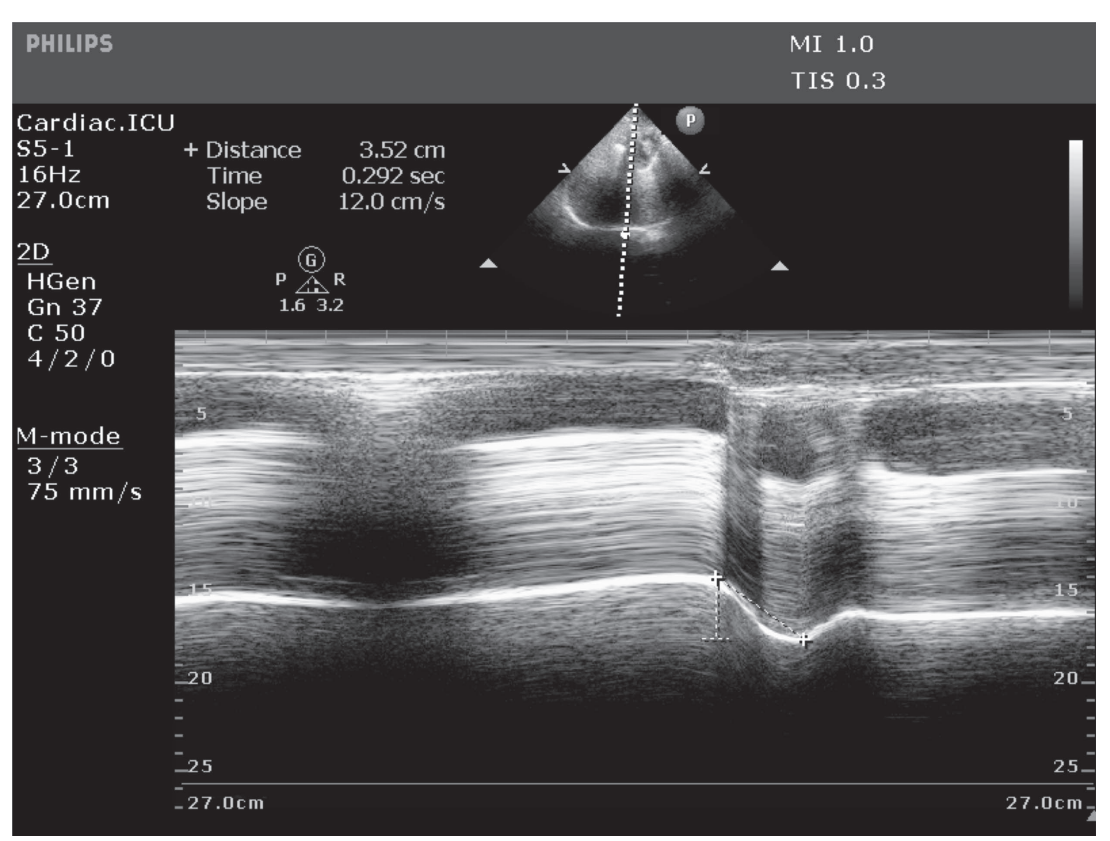

Fig. 2. Measurement of diaphragm excursion during the cough expiratory phase with the ultrasonographic M-mode.

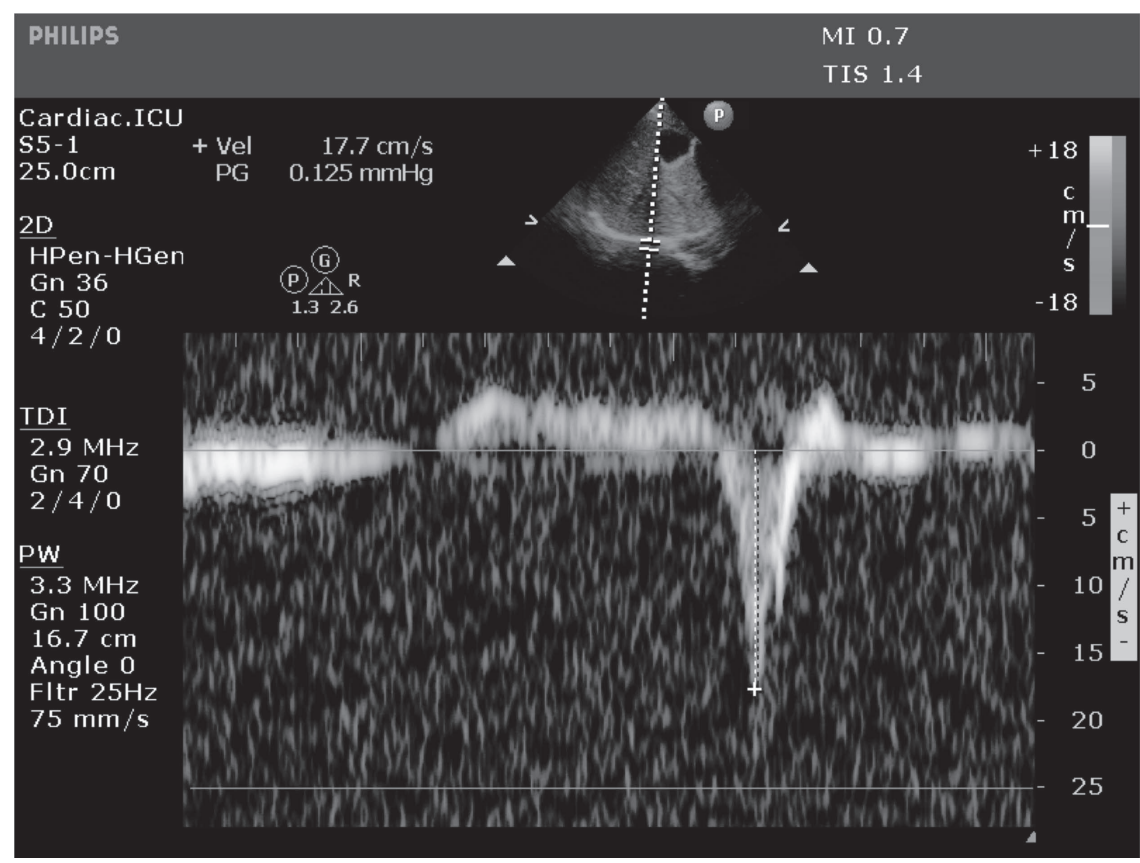

Fig. 3. Measurement of diaphragm peak velocity during the cough expiratory phase with the ultrasonographic tissue Doppler mode.

ment for sex, age, and height indicates that the relationship between $\mathrm{CPF}$ and diaphragmatic excursion is linked to physical demographics and thus diaphragmatic excursion should not be used as a single value to predict CPF.

A videofluoroscopic study reported that cephalic excursion was higher during involuntary cough (laryngeal cough expiratory reflex) than during voluntary cough; ${ }^{24}$ thus, ultrasonographic values likely have a different profile in
Table 2. Cough Peak Flow and Ultrasonographic Measurements

\begin{tabular}{lc}
\hline \hline Cough peak flow, L/s & $341.4 \pm 92$ \\
Diaphragm excursion, cm & $2.91 \pm 0.97$ \\
Diaphragm peak velocity, cm/s & $22.23 \pm 10.1$ \\
& \\
\hline Data are presented as mean \pm SD. & \\
\hline
\end{tabular}




\section{Diaphragm Excursion AND COUGH STRENGTH}

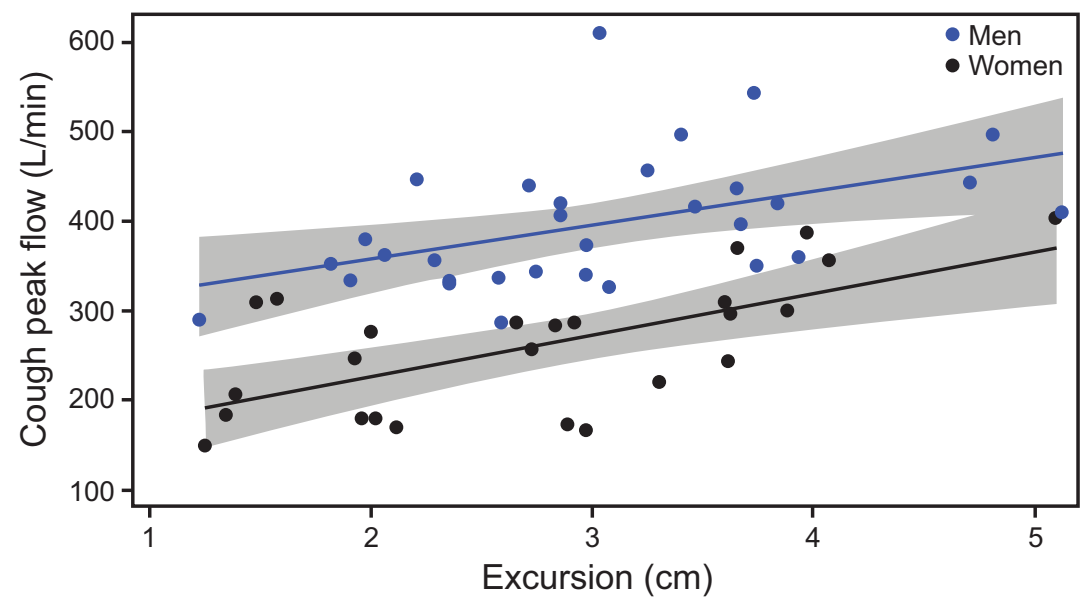

Fig. 4. Simple linear regression lines showing a positive correlation between diaphragm excursion and cough peak flow in men and women $\left(P<.001\right.$, beta coefficient 37.8, 95\% Cl 10.9-64.7, adjusted $\mathrm{R}^{2}=0.195$ for men; $P<.001$, beta coefficient $46.1,95 \% \mathrm{Cl} 22.3-69.9$, adjusted $\mathrm{R}^{2}=0.386$ for women). Shaded areas denote $95 \%$ Cls.

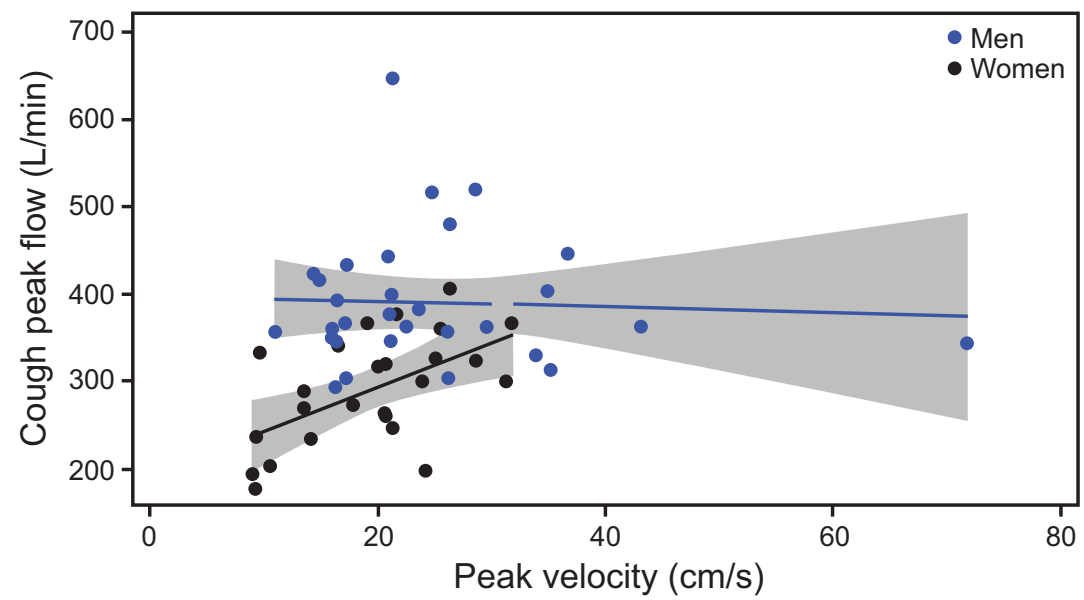

Fig. 5. Simple linear regression lines showing a positive correlation between diaphragm peak velocity and cough peak flow only in women $(P=.78$, beta coefficient $-0.33,95 \% \mathrm{Cl}-2.74$ to 2.08 for men; $P=.004$, beta coefficient $5.07,95 \% \mathrm{Cl} 1.81-8.33$ for women). Shaded areas denote $95 \%$ Cls.

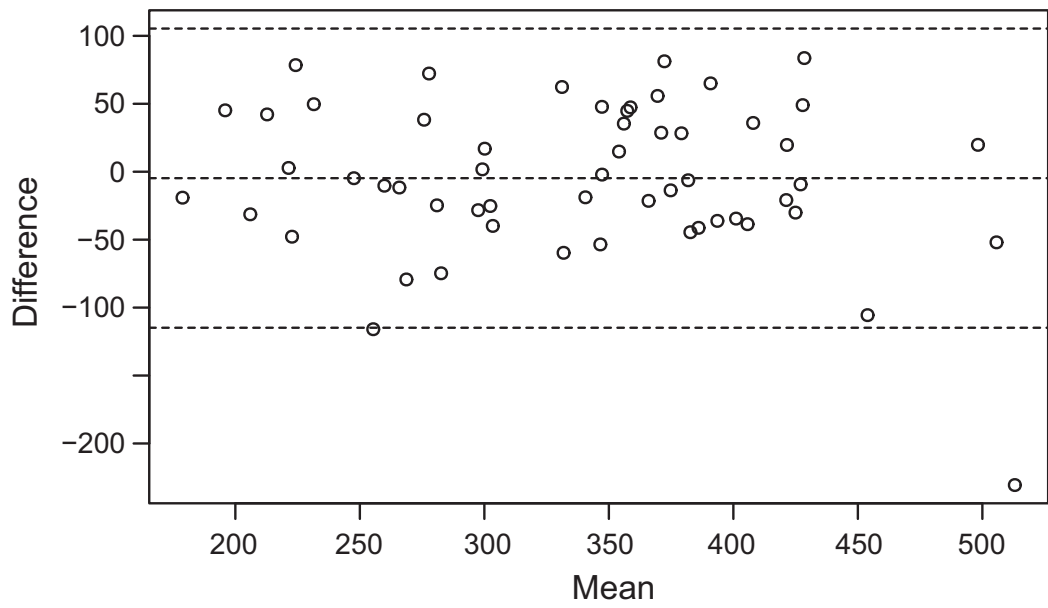

Fig. 6. Bland-Altman plot showing the difference between cough peak flow and predicted cough peak flow using diaphragm excursion, sex, height, and age. The center line shows the bias, and the upper and lower dashed lines denote bias \pm 2 SD $(n=56)$. 


\section{Diaphragm Excursion and Cough Strength}

involuntary cough. Future studies should investigate associations of ultrasonographic indices with CPF during involuntary cough. Interestingly, our study showed that diaphragm peak velocity was positively correlated with CPF in women but not in men. The reason for this sex difference is unclear, but these results need to be confirmed and validated in further studies.

The principal advantages of ultrasonographic measurement of cough strength are that it is simple and noninvasive. Therefore, our findings have potential application for evaluating cough strength in situations such as pre-extubation evaluation of mechanically ventilated patients and decision-making regarding whether to intubate patients with neuromuscular disorders who are at high risk of respiratory failure because of inadequate airway clearance.

This study has limitations that warrant mention. First, only healthy, relatively young volunteers who were not endotracheally intubated were studied. Second, diaphragm cough excursion and peak velocity are theoretically affected by respiratory system mechanics such as compliance of the lung and chest wall and airway resistance. In addition, characteristics of diaphragm movement during cough may differ substantially for patients with spinal cord injuries. ${ }^{25}$ Our results and ultrasonography data may not be generalizable to such patients. Future studies should investigate the relationship between cough strength and ultrasonography data in patients with various medical conditions, especially those on mechanical ventilation.

\section{Conclusion}

Passive cephalic excursion of the diaphragm during the cough expiratory phase significantly predicted $\mathrm{CPF}$ with maximum cough effort in healthy adults.

\section{ACKNOWLEDGEMENT}

We thank Professor Dimitris Georgopoulos for his helpful advice on this study.

\section{REFERENCES}

1. Wang ZY, Bai Y. Cough-another important factor in extubation readiness in critically ill patients. Crit Care 2012;16(6):461.

2. Norisue Y, Kataoka J, Homma Y, Naito T, Tsukuda J, Okamoto K, et al. Increase in intra-abdominal pressure during airway suctioninginduced cough after a successful spontaneous breathing trial is associated with extubation outcome. Ann Intensive Care 2018;8(1):61.

3. Laghi F, Tobin MJ. Disorders of the respiratory muscles. Am J Respir Crit Care Med 2003;168(1):10-48.

4. Black LF, Hyatt RE. Maximal respiratory pressures: normal values and relationship to age and sex. Am Rev Respir Dis 1969;99(5):696-702.

5. Man WD, Kyroussis D, Fleming TA, Chetta A, Harraf F, Mustfa N, et al. Cough gastric pressure and maximum expiratory mouth pressure in humans. Am J Respir Crit Care Med 2003;168(6):714-717.
6. Wilson SH, Cooke NT, Edwards RH, Spiro SG. Predicted normal values for maximal respiratory pressures in Caucasian adults and children. Thorax 1984;39(7):535-538.

7. Szeinberg A, Tabachnik E, Rashed N, McLaughlin FJ, England S, Bryan CA, et al. Cough capacity in patients with muscular dystrophy. Chest 1988;94(6):1232-1235.

8. Khamiees M, Raju P, DeGirolamo A, Amoateng-Adjepong Y, Manthous CA. Predictors of extubation outcome in patients who have successfully completed a spontaneous breathing trial. Chest 2001; 120(4):1262-1270.

9. Smina M, Salam A, Khamiees M, Gada P, Amoateng-Adjepong Y, Manthous CA. Cough peak flows and extubation outcomes. Chest 2003;124(1):262-268.

10. Duan J, Liu J, Xiao M, Yang X, Wu J, Zhou L. Voluntary is better than involuntary cough peak flow for predicting re-intubation after scheduled extubation in cooperative subjects. Respir Care 2014; 59(11):1643-1651.

11. Bai L, Duan J. Use of cough peak flow measured by a ventilator to predict re-intubation when a spirometer is unavailable. Respir Care 2017;62(5):566-571.

12. Beuret P, Roux C, Auclair A, Nourdine K, Kaaki M, Carton MJ. Interest of an objective evaluation of cough during weaning from mechanical ventilation. Intensive Care Med 2009;35(6):1090-1093.

13. Salam A, Tilluckdharry L, Amoateng-Adjepong Y, Manthous CA. Neurologic status, cough, secretions and extubation outcomes. Intensive Care Med 2004;30(7):1334-1339.

14. Su WL, Chen YH, Chen CW, Yang SH, Su CL, Perng WC, et al. Involuntary cough strength and extubation outcomes for patients in an ICU. Chest 2010;137(4):777-782.

15. Grelot L, Milano S. Diaphragmatic and abdominal muscle activity during coughing in the decerebrate cat. Neuroreport 1991;2(4):165-168.

16. McCool FD. Global physiology and pathophysiology of cough: ACCP evidence-based clinical practice guidelines. Chest 2006;129(1 Suppl): 48S-53S.

17. Irwin RS, Rosen MJ, Braman SS. Cough. A comprehensive review. Arch Intern Med 1977;137(9):1186-1191.

18. Park GY, Kim SR, Kim YW, Jo KW, Lee EJ, Kim YM, et al. Decreased diaphragm excursion in stroke patients with dysphagia as assessed by M-mode sonography. Arch Phys Med Rehabil 2015; 96(1):114-121.

19. Cohen E, Mier A, Heywood P, Murphy K, Boultbee J, Guz A. Diaphragmatic movement in hemiplegic patients measured by ultrasonography. Thorax 1994;49(9):890-895.

20. Palkar A, Narasimhan M, Greenberg H, Singh K, Koenig S, Mayo P, et al. Diaphragm excursion-time index: a new parameter using ultrasonography to predict extubation outcome. Chest 2018;153(5):1213-1220.

21. Boussuges A, Gole Y, Blanc P. Diaphragmatic motion studied by M-mode ultrasonography: methods, reproducibility, and normal values. Chest 2009;135(2):391-400

22. Ayoub J, Milane J, Targhetta R, Prioux J, Chamari K, Arbeille P, et al. Diaphragm kinetics during pneumatic belt respiratory assistance: a sonographic study in Duchenne muscular dystrophy. Neuromuscul Disord 2002;12(6):569-575.

23. Yamada Y, Ueyama M, Abe T, Araki T, Abe T, Nishino M, et al. Time-resolved quantitative analysis of the diaphragms during tidal breathing in a standing position using dynamic chest radiography with a flat panel detector system ("dynamic x-ray phrenicography"): initial experience in 172 volunteers. Acad Radiol 2017;24(4):393-400.

24. Stephens RE, Addington WR, Miller SP, Anderson JW. Videofluoroscopy of the diaphragm during voluntary and reflex cough in humans. Am J Phys Med Rehabil 2003;82(5):384.

25. Zhang X, Plow E, Ranganthan V, Huang H, Schmitt M, Nemunaitis $\mathrm{G}$, et al. Functional magnetic stimulation of inspiratory and expiratory muscles in subjects with tetraplegia. PM R 2016;8(7):651-659. 